JOURNAL OF THE

AMERICAN MATHEMATICAL SOCIETY

Volume 16, Number 1, Pages 1-17

S 0894-0347(02)00403-4

Article electronically published on August 27, 2002

\title{
MAXIMAL PROPERTIES OF THE NORMALIZED CAUCHY TRANSFORM
}

\author{
ALEXEI POLTORATSKI
}

\section{INTRODUCTION}

This note is devoted to one of the canonical objects of complex analysis, the Cauchy integral.

For any summable function $f$ on the unit circle $\mathbb{T}$ one can define its Cauchy integral $K f$ in the unit disk $\mathbb{D}$ as

$$
K f(z)=\int_{\mathbb{T}} \frac{f(\xi) d m(\xi)}{1-\bar{\xi} z},
$$

where $m$ is the normalized Lebesgue measure on $\mathbb{T}$. By the Fatou Theorem, $K f$ can also be defined on $\mathbb{T}$ by its non-tangential boundary values. After such an extension one can view the Cauchy integral as a "transform", i.e., an operator in $L^{p}(m)$ which sends $f$ into the boundary values of $K f$.

It is well known, and not difficult to see, that the Cauchy transform is unbounded for $p=1$ or $p=\infty$. However, the classical theorem by M. Riesz says that the Cauchy transform is bounded in $L^{p}$ when $1<p<\infty$. Further progress is due to R. Hunt, B. Muckenhoupt and R. Wheeden, who studied spaces with absolutely continuous weights $L^{p}(w)$. It was shown that the Cauchy transform is bounded in $L^{p}(w), 1<p<\infty$, if and only if the weight $w$ satisfies the celebrated $A_{p}$-condition.

Another classical object of complex function theory is the non-tangential maximal function. For any function $g$ in $\mathbb{D}$ we can define its non-tangential maximal function $M g$ as

$$
M g(\xi)=\sup _{z \in \Gamma_{\xi}}|g(z)|,
$$

where $\xi \in \mathbb{T}$ and $\Gamma_{\xi}$ is the sector $\left\{z \in \mathbb{T}|| z-\xi|\leq \sqrt{2} \operatorname{Re}(1-\bar{\xi} z)| z \mid,>\frac{1}{\sqrt{2}}\right\}$ (see Figure 1).

If $f$ is from $L^{p}(m)$ or from a weighted space $L^{p}(w)$, one can consider its Cauchy integral $K f$ and the maximal function $M K f$. After that one can, once again, view $M K$ as a transform in $L^{p}$. It is well known that in this regard $M K$ has exactly the same properties as $K$ : it is bounded in $L^{p}(m), 1<p<\infty$. Moreover, the analogy extends to the case of absolutely continuous weights: $M K$ is bounded in $L^{p}(w), 1<p<\infty$, iff $w$ satisfies $A_{p}$.

We refer the reader to $\mathrm{G}$ for the bibliography related to the above discussion.

Received by the editors June 12, 2000.

2000 Mathematics Subject Classification. Primary 30E20.

Key words and phrases. Cauchy integrals, boundary convergence, non-tangential maximal function.

The author is supported in part by N.S.F. grant DMS 9970151. 


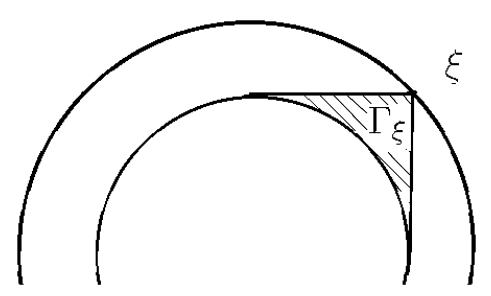

FiguRE 1.

These fundamental results on the Cauchy transform and the non-tangential maximal function play a prominent role in many areas of complex function theory. They provide useful information on the behavior of the Cauchy integrals in complex domains. Some of the most important applications of the Cauchy integrals lie in operator theory and mathematical physics. In these areas a Cauchy integral can appear as a resolvent function of an operator or, for instance, as a Weyl function related to a Schrödinger equation. In such applications, however, the corresponding weight (spectral measure) is rarely absolutely continuous, which makes the classical results insufficient. The purpose of this paper is to find an analog of the classical theory for the case of arbitrary weights.

Let $M(\mathbb{T})$ be the space of finite complex measures on the unit circle $\mathbb{T}$. We denote by $M_{+}(\mathbb{T})$ the subset consisting of positive measures. Let $\mu \in M_{+}(\mathbb{T})$ and $f \in L^{p}(\mu)$. Now, if we want to study $K f$ as in the classical theory, we have a slight problem: if, for example, $\mu$ is a singular measure, then $f$ may not be defined almost everywhere on $\mathbb{T}$, which makes the integration with respect to the Lebesgue measure in (1) impossible. The natural way out is to integrate with respect to $\mu$, i.e., to consider $K f \mu$ :

$$
K f \mu=\int_{\mathbb{T}} \frac{f(\xi) d \mu(\xi)}{1-\bar{\xi} z} .
$$

Now another question arises. If $\mu$ is, once again, singular, then the boundary values of $K f \mu$ can be infinite $\mu$-almost everywhere. To correct that, we have to normalize our Cauchy transform and consider $C_{\mu} f$ :

$$
C_{\mu} f=\frac{K f \mu}{K \mu}=\frac{\int_{\mathbb{T}} \frac{f(\xi) d \mu(\xi)}{1-\bar{\xi} z}}{\int_{\mathbb{T}} \frac{d \mu(\xi)}{1-\bar{\xi} z}} .
$$

In comparison with (1), we first include the measure $\mu$ in $K f \mu$, but then "factor it out" dividing by $K \mu$. Now we can ask all the canonical questions about the action of the normalized Cauchy transform $C_{\mu}$ and the associated maximal operator $M C_{\mu}$ in $L^{p}(\mu)$. These questions are the focus of the present paper.

The function-theoretical aspects of the normalized Cauchy transform were studied by D. Clark C], D. Sarason [S], A. Aleksandrov [A1]- A5] and the author [P1][P3]. The operator $C_{\mu}$ also appears in a number of applications, including problems in operator theory, mathematical physics and perturbation theory; see for instance C] or [P4]- $\mathrm{P} 6$.

First, we would like to present a brief survey of some known properties of this operator.

Let us look at the image of $L^{2}(\mu)$ under $C_{\mu}$. If $\mu=m$, then $C_{\mu}\left(L^{2}(\mu)\right)$ is the standard Hardy space $H^{2}$. If $\mu$ is a singular probability measure, we obtain the 
famous model space $K_{\theta}^{2}=H^{2} \ominus \theta H^{2}$, where $\theta$ is an inner function related to $\mu$ by the formula

$$
K \mu=\frac{1}{1-\theta}
$$

see $[\mathrm{C}]$. The spaces $K_{\theta}^{2}$ play a crucial role in operator model theory as the only invariant subspaces of the backward shift operator; see $[\mathrm{N}]$. Finally, when $\mu$ is an arbitrary measure having non-trivial absolutely continuous and singular parts, $C_{\mu}\left(L^{2}(\mu)\right)$ gives us yet another well-known space, the de Branges-Rovnyak space $\mathcal{M}_{\theta}^{2}$, where $\theta$ again satisfies (3), but now is not inner; see [S].

Here we will concern ourselves mostly with the second of these three examples. When $\mu$ is singular, $C_{\mu}$ not only maps $L^{2}(\mu)$ onto $K_{\theta}^{2}$, it actually preserves the norms [C] (the norm in $K_{\theta}^{2}$ is inherited from $H^{2}$ ). This beautiful fact can be viewed as an analog of Plancherel's formula and $C_{\mu}$ itself as a generalization of the Fourier transform. The connection between $C_{\mu}$ and $K_{\theta}^{2}$ provides most of the tools that will be used in this paper.

For any $\mu, C_{\mu}$ is bounded as an operator from $L^{2}(\mu)$ to $H^{2}$ (or to $L^{2}(m)$ ). The situation becomes more interesting for $p \neq 2$ :

Theorem 1 ([A2]). For any $\mu \in M_{+}(\mathbb{T})$ the operator $C_{\mu}$ is bounded as an operator from $L^{p}(\mu)$ to $H^{p}\left(\right.$ to $\left.L^{p}(m)\right)$ for $1<p \leq 2$. The operator $C_{\mu}$ is unbounded, in general, for $p>2$. In particular, if $\mu$ is singular and $C_{\mu}: L^{p}(\mu) \mapsto H^{p}\left(L^{p}(m)\right)$ is bounded for some $p>2$, then $\mu$ is discrete.

Now we can return to our main goal which is to study the boundedness of $C_{\mu}$ and $M C_{\mu}$ as transforms in $L^{p}(\mu)$. Let us start with $C_{\mu}$. First, we must establish the mechanism of the action of $C_{\mu}$. As in the classical case, $C_{\mu} f$ is an analytic function in $\mathbb{D}$ which has non-tangential boundary values a.e., on $\mathbb{T}$ with respect to the Lebesgue measure. But that does not mean that $C_{\mu} f$ has boundary values $\mu$-a.e., since $\mu$ is now allowed to have a non-trivial singular part. The following theorem takes care of this problem.

Theorem $2([\overline{\mathrm{P} 1}])$. Let $\mu \in M(\mathbb{T})$ and $f \in L^{1}(\mu)$. Then the function $C_{\mu} f$ has finite non-tangential boundary values $\mu$-a.e. These values are equal to $f \mu^{s}$-a.e., where $\mu^{s}$ is the singular component of $\mu$.

Now we know that $C_{\mu}$ can be naturally defined as a transform in $L^{p}(\mu)$. Moreover, the problem of its boundedness is solved for all $p \geq 1$ in the most difficult case of singular $\mu$. For such measures $C_{\mu}$ is not only bounded, it is identical. This reflects the fact that for singular $\mu$ the measure $f \mu$ is never "antianalytic".

For arbitrary $\mu$ the situation is not as nice. The same "forces" that make Theorem 1 fail for $p>2$ play a role here. All in all, we have the following:

Theorem 3. For any $\mu \in M_{+}(\mathbb{T})$ the operator $C_{\mu}$ is bounded in $L^{p}(\mu)$ for $1<p \leq$ 2 .

The operator is unbounded (in general) for $p>2$. As we will see from Example 1 in Section 3, the existence of measures $\mu$ such that $C_{\mu}$ is unbounded for $p>2$ follows from Theorem 1 . Unlike the case of singular $\mu$, the operator is not, generally, bounded when $p=1$; the simplest counterexample is $\mu=m$. In Section 4 we will prove Theorem 3.

Now let us discuss the action of the maximal operator $M C_{\mu} f$ in $L^{p}(\mu)$. This part contains the main new result of the paper. 
As usual, we say that an operator has weak type $(p, p)$ if it acts from $L^{p}(\mu)$ into "weak $L^{p}(\mu)$ ", defined as

$$
L^{p, \infty}(\mu)=\left\{f \mid \mu(\{|f|>t\})<\frac{C}{t^{p}}, \text { for some } C<\infty\right\}
$$

(with the inf of such $C$ raised to the power $1 / p$ viewed as a norm).

From Theorem 2 we know that $M C_{\mu} f$ is at least finite $\mu$-a.e. Also, as we will discuss in Section 4, one of the results of [P1] implies that $M C_{\mu}$ has weak type $(2,2)$. Until now nothing else was known about this maximal operator for any $p$. Here we present the following result.

Theorem 4. For any $\mu \in M_{+}(\mathbb{T})$ the maximal operator $M C_{\mu}$ is bounded in $L^{p}(\mu)$ for $1<p<2$.

The operator $M C_{\mu}$ is, generally, unbounded for $p>2$. In Section 3 we will construct an example of a singular $\mu$ and $f \in L^{\infty}(\mu)$ such that $M C_{\mu} f \notin L^{p}(\mu)$ for any $p>2$. The operator is obviously unbounded for $p=1$ as well. Hence, the only remaining question is whether weak type $(2,2)$ can be improved to the strong type. It seems that the answer is negative, but a counterexample is yet to be found. Apart from the boundedness, it would also be interesting to see if the operator has weak type $(1,1)$.

In the classical theory, as we discussed in the beginning of this introduction, the maximal operator always follows the pattern of the Cauchy transform itself: they are bounded under the same conditions. With the normalized Cauchy transform the situation is different. As we saw above, if we restrict our attention to the case of singular measures $\mu$, then the boundedness of $C_{\mu}$ will follow trivially from Theorem 2 for all $p \geq 1$. The maximal operator however will still be, generally, unbounded for $p>2$, as we will see from Example 2 in Section 3 .

A few final remarks. When trying to prove Theorem 4, the first natural idea is to apply standard estimates for analytic functions inside the disk such as the Schwarz Lemma, Harnack's Lemma, the maximal theorems mentioned in the beginning or their various corollaries. One quickly realizes however that all these results are based on the direct estimates of the Poisson kernel. Hence if such a proof could be found, it would also work for the operator

$$
f \mapsto M \frac{K f \mu}{P \mu},
$$

where $P \mu$ is the Poisson integral of $\mu$.

This observation brings up a few questions that seem interesting by themselves: is this operator bounded in $L^{p}(\mu)$ ? If not, does this operator have weak type $(p, p)$ or, at least, strong type $(p, p-\varepsilon)$ ? Example 3 (Section 3 ) shows that all the answers are negative: there exist $\mu \in M_{+}(\mathbb{T})$ and $f \in L^{\infty}(\mu)$ such that

$$
M \frac{K f \mu}{P \mu} \notin L^{p}(\mu)
$$

for any $p>0$. This illustrates the fact that our problem concerns singular integrals and cannot be treated with "non-singular" methods.

After that realization, the next natural idea is to apply classical "singular" tools such as the Calderon-Zygmund theory. The following objection immediately arises. If one replaces the Cauchy transform $K$ in the definition of our operator $C_{\mu}$ with the standard Hilbert transform $H$, understood in the sense of principal value, then 
Theorems 2-4 will no longer be valid. The easiest counterexample is $\mu=m+\delta_{1}$, where $\delta_{1}$ denotes the unit point mass at 1 , and $f$ defined as 1 on the upper halfcircle and as 0 elsewhere. Then $C_{\mu} f$ (defined with $H$ instead of $K$ ) is infinite at point 1, which has non-zero measure. At first sight this objection may not seem serious. We may try, for instance, to consider only continuous measures $\mu$ (without point masses). But new counterexamples can be found for this situation as well.

The same objection remains when one tries to apply the methods developed in the theory of Cauchy integrals in the plane (see for instance [D], [NTV], or [T]). The Cauchy integral considered there is, once again, the convolution with $\frac{1}{z}$ understood in the sense of principal value. Hence, the counterexample with a point mass, discussed above, still stands.

This difficulty in application of the known "singular" tools reflects the fact that our results describe a slightly different phenomenon. Theorems 2-4 hold true not because the singular part of the Cauchy transform (the conjugate Poisson integral) is "good" $\mu$-a.e., but because at $\mu$-a.e. point it is either "good" or "small" in comparison with the non-singular part (the Poisson integral). The non-singular part, in its turn, satisfies all the desired conditions and much more. The Hilbert transform as well as any other "purely singular" operator cannot be substituted into our theorems because it does not have such a non-singular part.

In the next section we introduce our main tools and make preparations for the proofs. In Section 3 we give all the announced counterexamples. Section 4 contains the proofs.

\section{Weighted LEVEL SETS OF INNER FUNCTIONS}

In the introduction we defined the "weak $L^{p "}$ spaces $L^{p, \infty}(\mathbb{T})$ for $\infty>p>0$. The corresponding "weak Hardy spaces" can now be defined as

$$
H^{p, \infty}=L^{p, \infty}(\mathbb{T}) \cap H^{q}, q<p,
$$

with the $L^{p, \infty}(\mathbb{T})$ norm.

We also introduced the space $K_{\theta}^{2}$ corresponding to an inner function $\theta$ as an orthogonal difference

$$
K_{\theta}^{2}=H^{2} \ominus \theta H^{2} .
$$

The space $K_{\theta}^{2}$ is a Hilbert space. It possesses a system of reproducing kernels $k_{\lambda}$ with the property that for any $\lambda \in \mathbb{D}$ and any $f \in K_{\theta}^{2}, f(\lambda)=\left\langle f, k_{\lambda}\right\rangle$. The kernels are defined as

$$
k_{\lambda}(z)=\frac{1-\overline{\theta(\lambda)} \theta(z)}{1-\bar{\lambda} z}
$$

The space $K_{\theta}^{p}, p>0$, is defined as the closed span of $k_{\lambda}$ 's in the topology of the Hardy space $H^{p}$. The weak space $K^{p, \infty}(\mathbb{T})$ can be defined as the intersection of $K_{\theta}^{q}$ for some $q<p$ and $H^{p, \infty}$.

Each function from the space $K_{\theta}^{p}\left(K_{\theta}^{p, \infty}\right)$ is known to possess the so-called "pseudocontinuation". Furthermore, when $p \geq 1$, the spaces $K_{\theta}^{p}$ are the only closed invariant subspaces of the backward shift operator $S^{*}: H^{p} \mapsto H^{p},\left(S^{*} f\right)(z)=$ $(f(z)-f(0)) / z$; see [N] for $p>1$ and [A6] for $p=1$. (When $p<1$, the spaces $K_{\theta}^{p}$ are not the only invariant subspaces of $S^{*}$; see [A6].)

A necessary and sufficient condition for a function $f$ to belong to $K_{\theta}^{p}\left(K_{\theta}^{p, \infty}\right)$ is that the function $\widetilde{f}$ defined on $\mathbb{T}$ as $\bar{f} \theta$ belongs to $H^{p}\left(H^{p, \infty}\right)$ and $\widetilde{f}(0)=0$. 
Let $\varphi \in H^{\infty}, \quad\|\varphi\|_{\infty} \leq 1$. Then for every $\alpha \in \mathbb{T}$ the function $(\alpha+\varphi)(\alpha-\varphi)^{-1}$ has positive real part in $\mathbb{D}$. Therefore there is a unique measure $\sigma_{\alpha}$ in $M_{+}(\mathbb{T})$ such that its Poisson integral satisfies

$$
P \sigma_{\alpha}=\int_{\mathbb{T}} \frac{\left(1-|z|^{2}\right) d \sigma_{\alpha}(\xi)}{|z-\xi|^{2}}=\operatorname{Re} \frac{\alpha+\varphi}{\alpha-\varphi} .
$$

In this way we can relate a family of measures $M_{\varphi}=\left\{\sigma_{\alpha}\right\}_{\alpha \in \mathbb{T}}$ to any function $\varphi$ from the unit ball of $H^{\infty}$.

Conversely, for each measure $\sigma$ in $M_{+}(\mathbb{T})$ there exists $\varphi \in H^{\infty}, \quad\|\varphi\|_{\infty} \leq 1$, such that $\sigma=\sigma_{1} \in M_{\varphi}$. Thus, the correspondence $\varphi \leftrightarrow \sigma_{1}$ (as well as any correspondence $\left.\varphi \leftrightarrow \sigma_{\alpha}, \alpha \in \mathbb{T}\right)$ provides a parametrization of the unit ball of $H^{\infty}$ by means of measures from $M_{+}(\mathbb{T})$.

Under this parametrization the subset of singular measures corresponds to the set of all inner functions. Indeed, suppose $\theta$ is inner. Then $(\alpha+\theta)(\alpha-\theta)^{-1}$ is pure imaginary a.e. on $\mathbb{T}$ and therefore all measures $\sigma_{\alpha} \in M_{\theta}$ are singular. Conversely, if $\sigma_{\alpha} \in M_{\theta}$ is singular for some $\alpha$, then $\theta$ is inner.

It is not hard to see that for inner $\theta$ the measure $\sigma_{\alpha} \in M_{\theta}$ is supported on the set of points where the non-tangential limits of $\theta$ are equal to $\alpha$. It follows that the measures $\sigma_{\alpha}$ are not only singular with respect to the Lebesgue measure, but also pairwise singular.

In the case of inner $\theta$ one can regard the measures $\sigma_{\alpha}$ as weighted level sets $\{\theta=\alpha\}$ of $\theta$. The weight reflects the speed of convergence of $\theta$ to its boundary value $\alpha$ at different points of $\mathbb{T}$. In particular, $\sigma_{\alpha}$ has a point mass at $\xi \in \mathbb{D}$ iff $\theta(\xi)=\alpha$ and $\theta$ has a non-tangential derivative at $\xi$, i.e., $\frac{\theta(z)-\alpha}{z-\xi}$ has a finite non-tangential limit at $\xi$ (see $[\underline{\mathrm{S}}]$ ).

The family $M_{\varphi}=\left\{\mu_{\alpha}\right\}_{\alpha \in \mathbb{T}}$ can be viewed as a decomposition of the normalized Lebesgue measure $m$ on $\mathbb{T}$. More precisely, the following integral formula holds (see [A1]):

$$
\int_{\mathbb{T}} \mu_{\alpha} d m(\alpha)=m
$$

This formula should be understood in the sense that any Lebesgue measurable set $E \subset \mathbb{T}$ is $\mu_{\alpha}$-measurable for $m$-a.e. $\alpha$ and $\int_{\mathbb{T}} \mu_{\alpha}(E) d m(\alpha)=m(E)$. In particular, if $\mathcal{E} \subset \mathbb{T}, \varphi$ is inner and $E=\varphi^{-1}(\mathcal{E})=\{\xi \in \mathbb{T} \mid \varphi(\xi) \in \mathcal{E}\}$, then

$$
m(E)=\int_{\mathcal{E}}\left\|\mu_{\alpha}\right\| d m(\alpha)
$$

If $\varphi(0)=0$, then all $\mu_{\alpha} \in M_{\varphi}$ are probability measures. If in addition $\varphi$ is inner, then (5) presents the well-known property that $\varphi$ preserves the Lebesgue measure on $\mathbb{T}$.

We will also use (4) in the following more general form. If $f$ is a summable function on $\mathbb{T}$, then it is $\mu_{\alpha}$-summable for a.e. $\alpha$ and

$$
\int_{\mathcal{E}} \int_{\mathbb{T}} f(\xi) d \mu_{\alpha}(\xi) d m(\alpha)=\int_{E} f(\xi) d m(\xi)
$$

for any sets $E, \mathcal{E}$ defined as above; see [A1].

As we discussed in the introduction, for any inner $\theta$

$$
K_{\theta}^{2}=C_{\sigma_{1}}\left(L^{2}\left(\sigma_{1}\right)\right)
$$


In this formula $\sigma_{1}$ can be replaced with any $\sigma_{\alpha}$. Theorem 2 now says that any $f \in K_{\theta}^{2}$ has non-tangential boundary values $\sigma_{\alpha}$-a.e. for any $\alpha \in \mathbb{T}$. These boundary values belong to $L^{2}\left(\sigma_{\alpha}\right)$. Moreover, $f$ can be recovered from its boundary values by means of $C_{\sigma_{\alpha}}$.

Here are a few more simple observations that we will need in our proofs. We denote by $P_{ \pm}$the standard Riesz projections.

Lemma 5. If $f \in K_{\theta}^{2}$, then:

1) $f^{2} \in K_{\theta^{2}}^{1}$.

2) The functions $u=\theta P_{-}\left(\bar{\theta} f^{2}\right)$ and $v=P_{+}\left(\bar{\theta} f^{2}\right)$ belong to $K_{\theta}^{1, \infty}$.

3) The function $f^{2}$ satisfies $f^{2}=u+\theta v$.

4) Let $M_{\theta}=\left\{\mu_{\alpha}\right\}_{\alpha \in \mathbb{T}}$. Then for any $\alpha \in \mathbb{T}$

$$
C_{\mu_{\alpha}} f^{2}=u+\alpha v \text {. }
$$

Also if $C_{\mu_{1}} f^{2}=C_{\mu_{\alpha}}$ g for some $g \in L^{1}\left(\mu_{\alpha}\right)$, then

$$
C_{\mu_{\alpha}}\left(g-f^{2}\right)=(1-\alpha) v .
$$

5) Let $\theta(0)=0$. Then all measures $\mu_{\alpha}$ are probability measures. If $M_{\theta^{2}}=$ $\left\{\sigma_{\alpha}\right\}_{\alpha \in \mathbb{T}}$, then $\sigma_{\alpha}$ 's can be derived from $\mu_{\alpha}$ 's through the following formula:

$$
\sigma_{\alpha}=1 / 2\left(\mu_{\sqrt{\alpha}}+\mu_{-\sqrt{\alpha}}\right)
$$

Proof. 1) Notice that the function $\bar{f}^{2} \theta^{2}$ belongs to $H^{1}$ and has value 0 at 0 .

2) $u, v \in H^{1, \infty}$ by the standard properties of the Riesz projectors. Now the statement follows from the fact that $\bar{\theta} u$ and $\bar{\theta} v$ are antianalytic.

3) Can be verified by simple calculations.

4) If $f$ is a bounded function, then $u, v$ and $C_{\mu_{\alpha}} f^{2}$ all belong to $K_{\theta}^{2}$. Moreover, $C_{\mu_{\alpha}} f^{2}$ and $u+\alpha v$ have the same boundary values $\mu_{\alpha}$-a.e. because $f^{2}=u+\theta v$ and $\theta=\alpha \mu_{\alpha}$-a.e. Since any $K_{\theta}^{2}$-function can be uniquely recovered from its boundary values in $L^{2}\left(\mu_{\alpha}\right),(7)$ holds. The general case follows from the fact that bounded functions are dense in $K_{\theta}^{2}$. To prove the next formula, just notice that $C_{\mu_{\alpha}} g=u+v$.

5) Follows from the formula

$$
K \sigma_{\alpha}=\frac{1}{1-\bar{\alpha} \theta^{2}}=\frac{1}{2}\left(\frac{1}{1-\overline{\sqrt{\alpha}} \theta}+\frac{1}{1-\overline{(-\sqrt{\alpha})} \theta}\right)=\frac{1}{2}\left(K \mu_{\sqrt{\alpha}}+K \mu_{-\sqrt{\alpha}}\right) .
$$

To conclude this section, let us mention that families $\left\{\sigma_{\alpha}\right\}_{\alpha \in \mathbb{T}}$ have many interesting properties and applications in complex analysis, functional analysis and perturbation theory. Such families were first introduced by D. Clark in $[\mathrm{C}]$ and further studied by D. Sarason [S], A. Aleksandrov [A1]- A5] and the author [P1]-[P6].

\section{Counterexamples}

Example 1. Our first example will show that the statement of Theorem 3 fails for $p>2$. As we will see, the existence of "bad" measures immediately follows from Theorem 1.

As was shown in [A2, if $\sigma \in M_{+}(\mathbb{T})$ is a non-discrete singular measure, then for any $p>2$ there exists $f \in L^{p}(\sigma)$ such that $C_{\sigma} f \notin H^{p}$. Put $\mu=\sigma+m$. Let us 
define the function $F$ :

$$
F(\xi)= \begin{cases}f(\xi), & \text { for } \sigma \text {-a.e. } \xi \in \mathbb{T} \\ 0, & \text { for } m \text {-a.e. } \xi \in \mathbb{T}\end{cases}
$$

Then $F \in L^{p}(\mu)$. But since $|K \sigma| \geq \frac{1}{2}|| \sigma \|$ in $\mathbb{D}$

$$
\left|C_{\mu} F\right|=\left|\frac{K f \mu}{K \mu}\right|=\left|\frac{K f \sigma}{K \sigma+1}\right| \geq \frac{\frac{1}{2}|| \sigma \|}{\frac{1}{2}|| \sigma||+1}\left|C_{\sigma} f\right| .
$$

Since $C_{\sigma} f \notin H^{p}, C_{\mu} F \notin L^{p}(\mu)$.

Example 2. Our next example will demonstrate the unboundedness of the maximal transform $M C_{\mu}$ for $p>2$. We will construct $\mu \in M_{+}(\mathbb{T})$ and $f \in L^{\infty}(\mu)$ such that $M C_{\mu} f \notin L^{p}(\mu)$ for any $p>2$.

Consider a sequence of points $\left\{a_{n}\right\}$ in $\mathbb{D}$ satisfying

$$
a_{n}=r_{n} e^{i \varphi_{n}}, \quad \varphi_{n}=\frac{1}{n}, \quad r_{n}=1-\frac{1}{n^{3} \ln ^{2}(n+1)} .
$$

Then

$$
\sum_{n} \frac{1-\left|a_{n}\right|^{2}}{\left|1-a_{n}\right|^{2}}<\sum_{n} \frac{\frac{1}{n^{3} \ln ^{2}(n+1)}}{1 / n^{2}}=\sum_{n} \frac{1}{n \ln ^{2}(n+1)}<\infty .
$$

Let $B$ be the Blaschke product with zeros at $\left\{a_{n}\right\}$ and at 0 . A theorem of Frostman F] says that any Blaschke product whose zeros satisfy

$$
\sum_{n} \frac{1-\left|a_{n}\right|^{2}}{\left|1-a_{n}\right|^{2}}<\infty
$$

has a non-tangential limit of absolute value 1 and finite non-tangential derivative at point 1 . Let $B(1)=\alpha$. As we discussed in the previous section, the existence of the non-tangential derivative at this point implies that the measure $\sigma_{\alpha} \in M_{B}$ has a point mass at 1 .

Put $\mu=\sigma_{\alpha}+m$ and define $f \in L^{\infty}(\mu)$ as 1 at point 1 and as 0 elsewhere. Then

$$
\left|C_{\mu} f\left(a_{n}\right)\right|=\left|\frac{\mu(\{1\}) \frac{1}{1-a_{n}}}{K \mu\left(a_{n}\right)}\right|=\left|\frac{\mu(\{1\}) \frac{1}{1-a_{n}}}{\frac{1}{1-\bar{\alpha} B}+1}\right|=\left|\frac{\mu(\{1\}) \frac{1}{1-a_{n}}}{2}\right| \geq C n
$$

for some $C>0$. For each $n$ consider an arc $I_{n} \subset \mathbb{T}$ centered at $\frac{a_{n}}{\left|a_{n}\right|}$ such that $\left|I_{n}\right|=\frac{1}{2 n^{3} \ln ^{2}(n+1)}$ (here and throughout the paper we often use the notation $|E|$ instead of $m(E))$. By (11) we have that for any $\xi \in I_{n}$

$$
M C_{\mu} f(\xi) \geq\left|C_{\mu} f\left(a_{n}\right)\right| \geq C n .
$$

Now let $p=2+\varepsilon, \varepsilon>0$. Then, since $I_{n}$ are disjoint,

$$
\left\|M C_{\mu} f\right\|_{L^{p}(\mu)}^{p} \geq\left\|M C_{\mu} f\right\|_{L^{p}(m)}^{p} \geq \sum_{n}\left|I_{n}\right|(C n)^{p}=\sum_{n} \frac{1}{2 n^{1-\varepsilon} \ln ^{2}(n+1)}=\infty .
$$

As one can see, in the above example $M C_{\mu} f$ might still belong to $L^{2}(\mu)$. It leaves open the question about the strong type $(2,2)$. While the answer is unknown, or if it is negative, it would also be interesting to verify if $M C_{\mu}$ is bounded as an operator from $L^{p}(\mu)$ to $L^{2}(\mu)$ for $p>2$ or at least for $p=\infty$. 
Example 3. This simple example illustrates the "singularity" of our problem. By Theorem 4 for any $\mu \in M_{+}(\mathbb{T}), f \in L^{p}(\mu), 1<p<2$, we have $M \frac{K f \mu}{K \mu} \in L^{p}(\mu)$. Here we will show that there exists a measure $\mu$ and a function $f \in L^{\infty}(\mu)$ such that

$$
M \frac{K f \mu}{P \mu} \notin L^{p}(\mu)
$$

for any $p>0$.

It is enough to show that for any $C>0$ there exist $\mu$ and $f \in L^{\infty}(\mu),\|f\|_{\infty} \leq 1$, such that

$$
\left\|M \frac{K f \mu}{P \mu}\right\|_{L^{p}(\mu)} \geq \frac{C}{2^{1 / p}}
$$

for any $p>0$. The existence of the desired example will follow by standard argument.

Consider the arc $I=\left\{e^{i \varphi} \mid \varepsilon<\varphi<1\right\}$, where the positive number $\varepsilon$ is chosen so small that

$$
\int_{I} \frac{1}{|1-\xi|} d m(\xi)>C
$$

Let $h(\xi)=\frac{1}{|1-\xi|}$ on $I$ and $h=0$ elsewhere. Consider the probability measure

$$
\mu=\frac{h m}{2\|h\|_{1}}+\frac{1}{2} \delta_{1},
$$

where $\delta_{1}$ is the unit point mass at 1 . Define $f \in L^{\infty}(\mu)$ as

$$
f(\xi)= \begin{cases}1, & \text { at } \xi=1, \\ 0, & \text { elsewhere. }\end{cases}
$$

Then for any $\xi \in I$

$$
M \frac{K f \mu}{P \mu}(\xi) \geq\left|\frac{K f \mu}{P \mu}(\xi)\right|=\|h\|_{1}>C .
$$

Therefore (12) holds for any $p>0$.

\section{Proofs}

We start with the proof of the maximal Theorem 4. First, we prove that $M C_{\mu}$ has weak type $(2,2)$; see Lemma 6 . Then we establish a weak-type estimate for the distribution function; see Lemma 13. After that the statement follows from a version of the Marcinkiewicz interpolation argument.

Lemma 6. For any $\mu \in M_{+}(\mathbb{T})$ the operator $M C_{\mu}$ has weak type $(2,2)$ (it is bounded as an operator from $L^{2}(\mu)$ to $\left.L^{2, \infty}(\mu)\right)$.

The proof is readily obtained from a result in [P1].

Proof. First let $\mu$ be a singular measure. Then $\mu=\mu_{1} \in M_{\theta}$ for some inner function $\theta$. As was shown in [P1], for every function $f$ from $K_{\theta}^{2}$

$$
\|M f\|_{L^{2, \infty}(\mu)} \leq C\|f\|_{H^{2}}
$$

for some absolute constant $C$. Since $C_{\mu}$ is a unitary operator from $L^{2}(\mu)$ onto $K_{\theta}^{2}$,

$$
\|f\|_{H^{2}}=\|f\|_{L^{2}(\mu)} .
$$




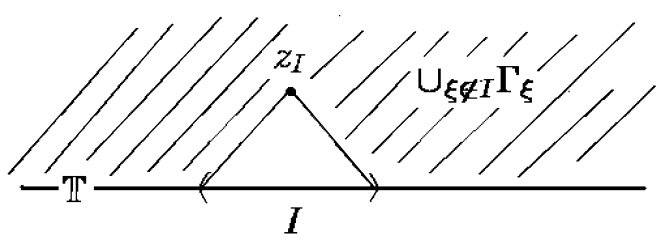

FigURE 2.

Together the last two formulas give us the statement.

If $\mu$ is an arbitrary positive measure, we can consider a sequence of singular measures tending to $\mu$ in the $*$-weak topology of the space of measures. Since the statement holds for each singular measure in the sequence and the constant $C$ in (13) is absolute, we can pass to the limit.

The case $p=1$ is much more complicated. The reason for that is the absence of Clark's Theorem. For $p<2$ the operator $C_{\mu}$ is not a surjection onto the corresponding $K_{\theta}^{p}$. Thus we cannot directly use the properties of pseudocontinuable functions from $K_{\theta}^{p}$ as in the proof of the weak type $(2,2)$ above.

Before we complete the proof of Theorem 4, we need some additional preparation.

Notation. Let $f$ be a function in $\mathbb{D}$ and $\Sigma$ a subset of $\mathbb{D}$. We denote by $M_{\Sigma} f$ the restricted maximal function:

$$
M_{\Sigma} f(\xi)=\sup _{z \in \Gamma_{\xi} \cap \Sigma}|f(z)|
$$

if $\Gamma_{\xi} \cap \Sigma \neq \emptyset$ and $M_{\Sigma} f(\xi)=0$ otherwise.

First let us notice that Lemma 6 yields the following

Corollary 7. Consider an inner function $\theta$ and the associated family of measures $M_{\theta}=\left\{\mu_{\alpha}\right\}$. Let $\alpha \in \mathbb{T}$ satisfy $\pi / 4<|\arg \alpha|<3 \pi / 4$. Denote

$$
\Sigma=\left\{z \in \mathbb{D} \mid P \mu_{1}>100\right\} .
$$

Then for any $f \in L^{2}\left(\mu_{\alpha}\right)$

$$
\left\|M_{\Sigma} K f \mu_{\alpha}\right\|_{L^{2, \infty}\left(\mu_{1}\right)} \leq D\|f\|_{L^{2}\left(\mu_{\alpha}\right)}
$$

for some absolute constant $D$.

Proof. Note that $|1-\bar{\alpha} \theta|>C>0$ on $\Sigma$. By Clark's Theorem the function $F=$ $C_{\mu_{\alpha}} f$ can also be represented as $F=C_{\mu_{1}} g$ for some $g \in L^{2}\left(\mu_{1}\right)$. Hence by Theorem 2 the non-tangential boundary values of $F$ exist and are equal to $g \mu_{1}$-a.e. Again, by Clark's Theorem $\|F\|_{H^{2}}=\|g\|_{L^{2}\left(\mu_{1}\right)}=\|f\|_{L^{2}\left(\mu_{\alpha}\right)}$. Now the statement follows from the fact that

$$
M_{\Sigma} K f \mu_{\alpha}=M_{\Sigma} \frac{1}{1-\bar{\alpha} \theta} C_{\mu_{\alpha}} f \leq M_{\Sigma} \frac{1}{C} C_{\mu_{1}} g
$$

and from Lemma 6 .

Notation. If $I \subset \mathbb{T}$ is an arc, we denote by $z_{I}$ the point in $\mathbb{D}$ such that $I$ is centered at $z_{I} /\left|z_{I}\right|$ and $I$ is seen from $z_{I}$ at the angle $\frac{\pi}{2}+2 \pi|I|$.

Note that if one considers the set $\mathbb{D} \backslash \bigcup_{\xi \notin I} \Gamma_{\xi}$ for $|I|<1 / 4$, it will consist of $\{|z| \leq 1 / \sqrt{2}\}$ and the "triangle" based on $I$ with the vertex at $z_{I}$; see Figure 2 . 
Lemma 8. Let $\sigma \in M_{+}(\mathbb{T}), C, p>0$ and $\Omega=\{z \in \mathbb{D} \mid P \sigma \leq C\}$. Then for any continuous function $F$ on $\mathbb{D}$

$$
\left\|M_{\Omega} F\right\|_{L^{p, \infty}(\sigma)} \leq D C\|M F\|_{L^{p, \infty}(m)}
$$

for some absolute constant $D>0$.

Proof. WLOG $M_{F} \in L^{p, \infty}(m)$. Fix $\lambda>0$ and put $E=\left\{\xi \in \mathbb{T} \mid M_{\Omega} F>\lambda\right\}$. We seek to bound $\sigma(E)$ by a constant multiple of $\frac{1}{\lambda^{p}}$. Consider a covering of $E$ by open $\operatorname{arcs} I$ such that $\left|F\left(z_{I}\right)\right|>(1-\epsilon) \lambda$ and $z_{I} \in \Omega$. Denote by $I_{1}, I_{2}, \ldots$ a subcovering of index 2 (each point is covered by at most two intervals). Since $P \sigma\left(z_{I_{n}}\right) \leq C$, $\sigma\left(I_{n}\right)<D_{0} C\left|I_{n}\right|$ and

$$
\sigma(E) \leq 2 \sum_{n} \sigma\left(I_{n}\right) \leq 2 D_{0} C\left|\bigcup_{n} I_{n}\right|
$$

Since $M F(\xi) \geq\left|F\left(z_{I_{n}}\right)\right|>(1-\epsilon) \lambda$ for all $\xi \in I_{n}$ and $M_{F} \in L^{p, \infty}(m)$, we get the desired bound.

Now we can use Lemma 8 to obtain

Corollary 9. Let $F$ be continuous in $\mathbb{D}$. Suppose that $M F \in L^{p, \infty}(m)$ for some $p>0$. Then for any $\sigma \in M_{+}(\mathbb{T})$ and any $C>0$

$$
\|M F\|_{L^{p, \infty}(\sigma)} \leq D\left(\left\|M_{\{|P \sigma|>C\}} F\right\|_{L^{p, \infty}(\sigma)}+\|M F\|_{L^{p, \infty}(m)}\right)
$$

for some absolute constant $D>0$.

Proof. The proof follows from Lemma 8 and the identity

$$
M F=\max \left(M_{\{|P \sigma|>C\}} F, M_{\{|P \sigma| \leq C\}} F\right) .
$$

Remark. For $F=\frac{K \nu}{K \mu}$ the inclusion $M F \in L^{p, \infty}(m)$ in the statement of Lemma 11 is automatic when $p \leq 1$. It may hold for $p>1$ if $\nu^{s}<<\mu^{s}$, which is the case we consider in Theorem 4 .

We will also need the following version of the weak type $(1,1)$ property of the Hardy-Littlewood maximal function.

Lemma 10. Let $\mu, \nu \in M(\mathbb{T}), \mu>0$. Then

$$
\left\|M \frac{P \nu}{P \mu}\right\|_{L^{1, \infty}(\mu)} \leq D\|\nu\|
$$

for some absolute constant $D$.

(Note that the estimate does not depend on $\|\mu\|$ because that quantity enters the left-hand side twice: in $P \mu$ and in the norm.)

Proof. WLOG $\nu$ is positive. Consider the Hardy-Littlewood maximal function:

$$
\mathcal{M}_{\nu, \mu}(\xi)=\sup \frac{\nu(I)}{\mu(I)}
$$

where sup is taken over all arcs $I$ containing $\xi$. By the standard argument $M \frac{P \nu}{P \mu} \leq$ $C_{1} \mathcal{M}_{\mu, \nu}$ for some absolute constant $C_{1}>0$. But $\mathcal{M}_{\nu, \mu} \in L^{1, \infty}(\mu)$; see for instance St], p. 44. 
A similar statement with $K \nu$ in the numerator instead of $P \nu$ is false as shown by Example 3. However, we still have the following weaker version.

Lemma 11. Let $\mu, \nu \in M(\mathbb{T}), \mu>0$. Then for any $\varepsilon>0$

$$
\left\|M \frac{K \nu}{(1+P \mu)^{1+\varepsilon}}\right\|_{L^{1, \infty}(\mu)} \leq D\|\nu\|
$$

for some constant $D$ depending only on $\varepsilon$.

Proof. WLOG $\nu$ is a positive measure. Then $K \nu$ is outer and we can consider the analytic function $f=(K \nu)^{\frac{1}{1+\varepsilon}}$. The function $f$ will belong to $H^{1+\varepsilon, \infty}$. The operator

$$
g \mapsto M \frac{P g m}{1+P \mu}
$$

acts from $L^{\infty}(m)$ to $L^{\infty}(\mu)$. By the last lemma it also acts from $L^{1}(m)$ to $L^{1, \infty}(\mu)$. Therefore by the Marcinkiewicz Interpolation Theorem it acts from $L^{1+\varepsilon, \infty}(m)$ to $L^{1+\varepsilon, \infty}(\mu)$. Hence

$$
\begin{aligned}
& \left\|M \frac{K \nu}{(1+P \mu)^{1+\varepsilon}}\right\|_{L^{1, \infty}(\mu)}=\left\|\left(M \frac{f}{1+P \mu}\right)^{1+\varepsilon}\right\|_{L^{1, \infty}(\mu)} \\
= & \left\|\left(M \frac{P f m}{1+P \mu}\right)^{1+\varepsilon}\right\|_{L^{1, \infty}(\mu)} \leq D_{1}\left(\|f\|_{L^{1+\varepsilon, \infty}(m)}\right)^{1+\varepsilon} \leq D_{2}\|\nu\| .
\end{aligned}
$$

Let $f$ be a function in $\mathbb{D}$ and $\mu$ a measure on $\mathbb{T}$. As before, when we say that $f \in L^{p}(\mu)$, we mean that the non-tangential boundary values of $f$ exist $\mu$-a.e. and belong to $L^{p}(\mu)$. When we write $f=C_{\mu} f$, we imply that $f \in L^{1}(\mu)$ and that $f$ can be recovered from its boundary values via $C_{\mu}$.

Lemma 12. Let $f \in K_{\theta}^{p}$ for some inner $\theta$ and $p \geq 1$. Consider the family of measures $\left\{\mu_{\alpha}\right\}=M_{\theta}$ associated with $\theta$. Then $f=C_{\mu_{\alpha}} f$ for almost every $\alpha \in \mathbb{T}$.

Proof. If $p \geq 2$, then $f=C_{\mu_{\alpha}} f$ for every $\alpha \in \mathbb{T}$ by Clark's Theorem and Theorem 2 (we already used this fact in earlier proofs). If $p<2$, consider a sequence of $K_{\theta}^{2}$-functions $f_{n}$ tending to $f$ in $H^{p}$. The formula (4) now implies that $f_{n} \rightarrow f$ in $L^{p}\left(\mu_{\alpha}\right)$ for a.e. $\alpha$ and the statement follows.

Problem. It would be interesting to understand if in the statement of Lemma 12 "almost every $\alpha$ " can be replaced with "every $\alpha$, such that the boundary values of $f$ exist $\mu_{\alpha}$-a.e.".

If $\mu \in M_{+}(\mathbb{T})$ and $f$ is a $\mu$-measurable function, we denote by $m_{f}^{\mu}$ its distribution function:

$$
m_{f}^{\mu}(\lambda)=\mu(\{|f|>\lambda\}) .
$$

To finish the proof of Theorem 4, we will need the following Marcinkiewicz-type estimate. 
Lemma 13. Let $f \in K_{\theta}^{p}$ for some inner $\theta$ and $p>0$. Consider the family of measures $\left\{\mu_{\alpha}\right\}=M_{\theta}$ corresponding to $\theta$. Suppose that $f=C_{\mu_{1}} f=C_{\mu_{\alpha}} f=C_{\mu_{-\alpha}} f$ for some $\alpha \in \mathbb{T}, \pi / 4<\arg \alpha<3 \pi / 4$. Denote $\Sigma=\left\{z \in \mathbb{D} \mid P \mu_{1}(z)>100\right\}$. Consider functions $g$ and $h$ defined $\left(\mu_{1}+\mu_{\alpha}+\mu_{-\alpha}\right)$-almost everywhere on the circle as

$$
g= \begin{cases}f, & \text { when }|f|<\lambda \\ 0, & \text { elsewhere }\end{cases}
$$

and

$$
h= \begin{cases}f, & \text { when }|f|>\lambda, \\ 0, & \text { elsewhere. }\end{cases}
$$

Then the restricted maximal function $M_{\Sigma} f$ satisfies

$$
m_{M_{\Sigma} f}^{\mu_{1}}(\lambda) \leq D\left(\frac{1}{\lambda^{2}}\|g\|_{L^{2}\left(\mu_{1}+\mu_{\alpha}+\mu_{-\alpha}\right)}+\frac{1}{\lambda}\|h\|_{L^{1}\left(\mu_{1}+\mu_{\alpha}+\mu_{-\alpha}\right)}\right)
$$

for some absolute constant $D$.

Proof. Since

$$
M_{\Sigma}\left(C_{\mu_{1}} f\right) \leq M_{\Sigma}\left(C_{\mu_{1}} h\right)+M_{\Sigma}\left(C_{\mu_{1}} g\right)
$$

and

$$
\left\|M\left(C_{\mu_{1}} g\right)\right\|_{L^{2, \infty}\left(\mu_{1}\right)}<C_{1}\|g\|_{L^{2}\left(\mu_{1}\right)}
$$

by Lemma 6 , it is left to estimate $M_{\Sigma}\left(C_{\mu_{1}} h\right)$.

Let $k$ be a function in $L^{2}\left(\mu_{1}\right)$ such that $k^{2}=h \mu_{1}$-a.e. Then by Lemma 5

$$
\left(C_{\mu_{1}} k\right)^{2}=u+\theta v
$$

for some $u, v \in K_{\theta}^{1, \infty}$. Also by Lemma 5 we have that $C_{\mu_{1}} h=u+v$. Therefore

$$
M\left(C_{\mu_{1}} h\right) \leq M(u+\theta v)+M((1-\theta) v) .
$$

Since

$$
\|M(u+\theta v)\|_{L^{1, \infty}\left(\mu_{1}\right)}=\left\|M\left(C_{\mu_{1}} k\right)^{2}\right\|_{L^{1, \infty}\left(\mu_{1}\right)} \leq C_{2}\|k\|_{L^{2}\left(\mu_{1}\right)}^{2},
$$

to finish the proof we need to estimate $(1-\theta) v$.

Note that by Lemma 5

$$
\begin{aligned}
v & =\frac{1}{1 \mp \alpha} C_{\mu_{ \pm \alpha}}\left(C_{\mu_{1}} h-\left(C_{\mu_{1}} k\right)^{2}\right)=\frac{1}{1 \mp \alpha} C_{\mu_{ \pm \alpha}}\left(C_{\mu_{1}}(f-g)-(u+\theta v)\right) \\
& =\frac{1}{1 \mp \alpha} C_{\mu_{ \pm \alpha}}\left(f-C_{\mu_{1}} g-(u+\theta v)\right)=\frac{1}{1 \mp \alpha} C_{\mu_{ \pm \alpha}}\left(h+g-C_{\mu_{1}} g-(u+\theta v)\right) .
\end{aligned}
$$

In other words, by Theorem 2

$$
v=\frac{1}{1 \mp \alpha}\left(h+g-C_{\mu_{1}} g-(u+\theta v)\right) \quad \mu_{ \pm \alpha} \text {-a.e. }
$$

and $C_{\mu_{ \pm \alpha}} v=v$.

Recalling the definition of $C_{\mu_{ \pm \alpha}} v$, we obtain

$$
\begin{aligned}
K v \mu_{\alpha} & =K \mu_{\alpha} C_{\mu_{\alpha}} v=\frac{v}{1-\bar{\alpha} \theta}=\frac{1+\bar{\alpha} \theta}{1-\bar{\alpha} \theta} \frac{v}{1+\bar{\alpha} \theta} \\
& =\frac{1+\bar{\alpha} \theta}{1-\bar{\alpha} \theta} K \mu_{-\alpha} C_{\mu_{-\alpha}} v=\frac{1+\bar{\alpha} \theta}{1-\bar{\alpha} \theta} K v \mu_{-\alpha} .
\end{aligned}
$$


Using the identity

$$
K \mu=1 / 2(P \mu+i Q \mu+1),
$$

we get

$$
P v \mu_{\alpha}+i Q v \mu_{\alpha}+v(0)=\frac{1+\bar{\alpha} \theta}{1-\bar{\alpha} \theta}\left(P v \mu_{-\alpha}+i Q v \mu_{-\alpha}+v(0)\right) .
$$

Since

$$
\frac{1+\bar{\alpha} \theta}{1-\bar{\alpha} \theta}=\frac{1+\bar{\alpha}}{1-\bar{\alpha}}-\frac{2 \bar{\alpha}}{(1-\bar{\alpha})(1-\bar{\alpha} \theta)}(1-\theta)
$$

and $1-\bar{\alpha} \theta$ is bounded away from 0 on $\Sigma,(18)$ yields

$$
\begin{aligned}
\quad\left|i Q v \mu_{\alpha}+v(0)+\frac{1+\bar{\alpha}}{1-\bar{\alpha}}\left(i Q v \mu_{-\alpha}+v(0)\right)\right| \\
\leq C_{3}\left(\left|P v \mu_{-\alpha}\right|+\left|P v \mu_{\alpha}\right|+|1-\theta|\left(\left|Q v \mu_{-\alpha}\right|+|v(0)|\right)\right) \text { on } \Sigma .
\end{aligned}
$$

Consider the family of measures $\left\{\sigma_{\beta}\right\}=M_{\theta^{2}}$ corresponding to the inner function $\theta^{2}$. By (9) we have $\sigma_{\alpha^{2}}=1 / 2\left(\mu_{\alpha}+\mu_{-\alpha}\right)$. Since both functions $v$ and $\bar{\alpha} \theta v$ belong to $K_{\theta^{2}}^{1, \infty}$ and $C_{\mu_{ \pm \alpha}} v=v$, one can easily verify that $v=C_{\sigma_{\alpha^{2}}} v$ and $\bar{\alpha} \theta v=C_{\sigma_{\alpha^{2}}} \bar{\alpha} \theta v$. Thus we obtain

$$
K v\left(\mu_{\alpha}+\mu_{-\alpha}\right)=2 C_{\sigma_{\alpha^{2}}} v K \sigma_{\alpha^{2}}=2 \frac{v}{1-\bar{\alpha}^{2} \theta^{2}}
$$

and, using the fact that $\theta= \pm \alpha \mu_{ \pm \alpha}$-a.e.,

$$
K v\left(\mu_{\alpha}-\mu_{-\alpha}\right)=2 C_{\sigma_{\alpha^{2}}} \bar{\alpha} \theta v K \sigma_{\alpha^{2}}=2 \frac{\bar{\alpha} \theta v}{1-\bar{\alpha}^{2} \theta^{2}} .
$$

Using these representations and the fact that $1-\bar{\alpha}^{2} \theta^{2}$ is bounded away from 0 on $\Sigma$, the left-hand side of (19) can be estimated as follows:

$$
\begin{aligned}
\left|i Q v \mu_{\alpha}+v(0)+\frac{1+\bar{\alpha}}{1-\bar{\alpha}}\left(i Q v \mu_{-\alpha}+v(0)\right)\right| \\
=\mid \frac{1}{1-\bar{\alpha}}\left(i Q v\left(\mu_{\alpha}+\mu_{-\alpha}\right)+v(0)\right) \\
\quad-\frac{\bar{\alpha}}{1-\bar{\alpha}}\left(i Q v\left(\mu_{\alpha}-\mu_{-\alpha}\right)+\bar{\alpha} \theta(0) v(0)\right)+\frac{1}{1-\bar{\alpha}} v(0) \mid \\
=\left|\frac{1}{1-\bar{\alpha}}\right| \mid\left(2 K v\left(\mu_{\alpha}+\mu_{-\alpha}\right)-P v\left(\mu_{\alpha}+\mu_{-\alpha}\right)\right) \\
\quad-\bar{\alpha}\left(2 K v\left(\mu_{\alpha}-\mu_{-\alpha}\right)-P v\left(\mu_{\alpha}-\mu_{-\alpha}\right)\right)+v(0) \mid \\
\geq C_{4}|v|-C_{5}\left(\left|P v \mu_{\alpha}\right|+\left|P v \mu_{-\alpha}\right|+|v(0)|\right) \text { on } \Sigma .
\end{aligned}
$$

Now, (19) and (22) give

$$
|v| \leq C_{6}\left(\left|P v \mu_{\alpha}\right|+\left|P v \mu_{-\alpha}\right|+|1-\theta|\left|Q v \mu_{-\alpha}\right|+|v(0)|\right) \quad \text { on } \Sigma .
$$

Multiplying (23) by $|1-\theta|$, we obtain

$$
\begin{aligned}
|(1-\theta) v| \leq C_{6}(\mid & (1-\theta) P v \mu_{\alpha}|+|(1-\theta) P v \mu_{-\alpha} \mid \\
& \left.+\left|(1-\theta)^{2} Q v \mu_{-\alpha}\right|+|(1-\theta) v(0)|\right) \text { on } \Sigma .
\end{aligned}
$$


If we note that $|1-\theta| \leq 2 \frac{1}{1+P \mu_{1}}$ and recall the representation (16) for $v$, we can transform (24) into

$$
\begin{aligned}
|(1-\theta) v| \leq C_{6}[ & \frac{\left|P(h-u-\theta v)\left(\mu_{\alpha}+\mu_{-\alpha}\right)\right|}{1+P \mu_{1}} \\
& +\frac{\left|P\left(g-C_{\mu_{1}} g\right)\left(\mu_{\alpha}+\mu_{-\alpha}\right)\right|}{1+P \mu_{1}}+\frac{\left|Q(h-u-\theta v) \mu_{-\alpha}\right|}{\left(1+P \mu_{1}\right)^{2}} \\
& \left.+\frac{\left|Q\left(g-C_{\mu_{1}} g\right) \mu_{-\alpha}\right|}{\left(1+P \mu_{1}\right)^{2}}+\frac{|v(0)|}{1+P \mu_{1}}\right] \text { on } \Sigma .
\end{aligned}
$$

Now we can estimate the maximal function $M_{\Sigma}$ of the right-hand side of (25). The first summand can be estimated using Lemma 10 together with the fact that $h$ is from $L^{1}\left(\mu_{1}+\mu_{\alpha}+\mu_{-\alpha}\right)$ and $\|u+\theta v\|_{L^{1}\left(\mu_{ \pm \alpha}\right)}=\|h\|_{L^{1}\left(\mu_{1}\right)}$. The second summand can be handled with Corollary 7 because $g$ is from $L^{2}\left(\mu_{1}+\mu_{\alpha}+\mu_{-\alpha}\right),\left\|C_{\mu_{1}} g\right\|_{L^{2}\left(\mu_{ \pm \alpha}\right)}=$ $\|g\|_{L^{2}\left(\mu_{1}\right)}$ by Clark's Theorem and $1+P \mu_{1} \geq 1$. The third summand can be estimated using Lemma 11. To the fourth summand we can, once again, apply Corollary 7 . Finally, the last summand can be easily estimated using the fact that $v(0)=\int v d \mu_{\alpha}$ together with (16). After showing that maximal functions of all summands belong to $L^{2, \infty}\left(\mu_{1}\right)$ or to $L^{1, \infty}\left(\mu_{1}\right)$ with norms that can be estimated through $\|g\|_{L^{2}\left(\mu_{1}+\mu_{\alpha}+\mu_{-\alpha}\right)}$ and $\|h\|_{L^{1}\left(\mu_{1}+\mu_{\alpha}+\mu_{-\alpha}\right)}$, respectively, we can conclude that the distribution function of $M_{\Sigma}(1-\theta) v$ satisfies

$$
m_{M_{\Sigma}(1-\theta) v}^{\mu_{1}}(\lambda) \leq C_{7}\left(\frac{1}{\lambda^{2}}\|g\|_{L^{2}\left(\mu_{1}+\mu_{\alpha}+\mu_{-\alpha}\right)}^{2}+\frac{1}{\lambda}\|h\|_{L^{1}\left(\mu_{1}+\mu_{\alpha}+\mu_{-\alpha}\right)}\right)
$$

for some absolute constant $C_{7}$.

Together with (14), (15) and the fact that $C_{\mu_{1}} f=C_{\mu_{1}} g+(u+\theta v)+(1-\theta) v$ we obtain the statement.

Remark. Instead of 1 and $\pm \alpha$ in the statement of Lemma 13 we could, of course, consider any three different indices from $\mathbb{T}$. In that case we would have to replace $\theta^{2}$ in the proof with $B(\theta)$, where $B$ is a properly chosen Blaschke product of order 2. The constant $D$ in the statement would then depend on the distances between the indices.

Now we are ready to prove Theorem 4.

Proof of Theorem 4. Suppose that $F \in L^{p}(\mu)$ for some $1<p<2$. WLOG $\mu$ is a probability measure. First, consider the case of singular $\mu$. Again let $\theta$ be an inner function such that $K \mu=\frac{1}{1-\theta}$. In other words, $\mu=\mu_{1} \in M_{\theta}=\left\{\mu_{\alpha}\right\}_{\alpha \in \mathbb{T}}$. Since $\|\mu\|=1, \theta(0)=0$.

Since $F \in L^{p}(\mu)$, by Theorem $1, f=C_{\mu} F \in H^{p}$. Since by Theorem $2, f=F$ $\mu$-a.e., in the rest of the proof we will write $f$ in place of $F$. By $(6) f \in L^{p}\left(\mu_{\alpha}\right)$ for a.e. $\alpha$ and

$$
C_{1}\|f\|_{L^{p}(\mu)}^{p}>\|f\|_{p}^{p}=\int_{\mathbb{T}}\|f\|_{L^{p}\left(\mu_{\alpha}\right)}^{p} d m(\alpha) .
$$

By Lemma 12 and (26) we can choose $\alpha$ such that $\pi / 4<\arg \alpha<3 \pi / 4$,

$$
f=C_{\mu_{ \pm \alpha}}(f)
$$

and

$$
\|f\|_{L^{p}\left(\mu_{ \pm \alpha}\right)}<C_{2}\|f\|_{L^{p}(\mu)} .
$$


Consider functions $g$ and $h$ and a set $\Sigma$ defined in the same way as in the statement of Lemma 13. Denote $\eta=\mu_{1}+\mu_{\alpha}+\mu_{-\alpha}$. Then by Lemma 13 the restricted maximal function $M_{\Sigma} f$ satisfies

$$
m_{M_{\Sigma} f}^{\mu_{1}}(\lambda) \leq D\left(\frac{1}{\lambda^{2}}\|g\|_{L^{2}(\eta)}+\frac{1}{\lambda}\|h\|_{L^{1}(\eta)}\right) .
$$

Now we can proceed as in the standard proof of the Marcinkiewicz Theorem to show that $M_{\Sigma} f \in L^{p}(\mu)$. Using the properties of the distribution function and the Fubini Theorem, we obtain

$$
\begin{aligned}
\int_{\mathbb{T}}\left|M_{\Sigma} f\right|^{p} d \mu_{1} & =p \int_{0}^{\infty} \lambda^{p-1} m_{M_{\Sigma} f}^{\mu_{1}}(\lambda) d \lambda \\
& \leq D p \int_{0}^{\infty} \lambda^{p-1}\left(\frac{1}{\lambda^{2}}\|g\|_{L^{2}(\eta)}^{2}+\frac{1}{\lambda}\|h\|_{L^{1}(\eta)}\right) d \lambda \\
& =D p \int_{0}^{\infty} \lambda^{p-1}\left(-\frac{1}{\lambda^{2}} \int_{0}^{\lambda} x^{2} d m_{f}^{\eta}(x)-\frac{1}{\lambda} \int_{\lambda}^{\infty} x d m_{f}^{\eta}(x)\right) d \lambda \\
& =D p\left(-\int_{0}^{\infty} x^{p} \int_{x}^{\infty} \lambda^{p-3} d \lambda d m_{f}^{\eta}(x)-\int_{0}^{\infty} x^{p} \int_{0}^{x} \lambda^{p-2} d \lambda d m_{f}^{\eta}(x)\right) \\
& =D p\left(-\frac{1}{2-p} \int_{0}^{\infty} x^{p} d m_{f}^{\eta}(x)-\frac{1}{p-1} \int_{0}^{\infty} x^{p} d m_{f}^{\eta}(x)\right) \\
& D p\left(\frac{\|f\|_{L^{p}(\eta)}}{2-p}+\frac{\|f\|_{L^{p}(\eta)}}{p-1}\right) .
\end{aligned}
$$

To pass from $M_{\Sigma} f$ to $M f$, notice that since $f=C_{\mu} F \in H^{p}$, we can apply Corollary 9 and obtain an estimate for $\|M f\|_{L}^{p, \infty}(\mu)$. The Marcinkiewicz Interpolation Theorem now implies that the maximal operator is bounded for every $q, p<q<2$. Since $p$ was arbitrary between 1 and 2, this gives us the statement. Similarly to the proof of Lemma 6 , from the case of singular $\mu$ one can pass to the general case.

Our last remaining proof follows from Theorem 4 and a result from [P1].

Proof of Theorem 3. The case $p=2$, once again, can be handled with the methods from [P1] because one has an opportunity to use Clark's Theorem. In fact, a more general result is proved in [P1]: if $f \in L^{2}(\mu)$, then the Fourier series $\sum_{n \geq 0} a_{n} z^{n}$ of the function $C_{\mu} f$ converges in $L^{2}(\mu)$. The limit of the Fourier series obviously coincides with the boundary values of $C_{\mu} f$ almost everywhere with respect to the absolutely continuous part of $\mu$. In addition, by Theorem 2 , the boundary values of $C_{\mu} f$ are equal to $f$ almost everywhere with respect to the singular part of $\mu$. Hence $C_{\mu} f \in L^{2}(\mu)$.

The rest follows from Theorem 4 .

\section{ACKNOWLEDGMENT}

The author would like to thank A. B. Aleksandrov for questions and useful discussions. 


\section{REFERENCES}

[A1] A. B. Aleksandrov, Multiplicity of boundary values of inner functions, Izv. Acad. Nauk. Arm. SSR, Matematica 225 (1987), 490-503 (Russian). MR 89e:30058

[A2] A. B. Aleksandrov, Inner functions and related spaces of pseudocontinuable functions, Zap. Nauchn. Sem. Leningrad. Otdel. Mat. Inst. Steklov. (LOMI) 170 (1989), 7-33 (Russian). MR 91c:30063

[A3] A. B. Aleksandrov, On the existence of angular boundary values for pseudocontinuable functions, Zap. Nauchn. Sem. S.-Peterburg. Otdel. Mat. Inst. Steklov. (POMI) 222 (1995) (Russian). MR 97a:30046

[A4] A. B. Aleksandrov, Isometric embeddings of coinvariant subspaces of the shift operator, Zap. Nauchn. Sem. S.-Peterburg. Otdel. Mat. Inst. Steklov. (POMI) 232 (1996) (Russian). MR 98k:30050

[A5] A. B. Aleksandrov, On the maximum principle for pseudocontinuable functions, Zap. Nauchn. Sem. S.-Peterburg. Otdel. Mat. Inst. Steklov. (POMI) 217 (1994) (Russian). MR 96c:30033

[A6] A. B. Aleksandrov, Invariant subspaces of the backward shift operator in the space $H^{p}$ $(p \in(0,1))$, Zap. Nauchn. Sem. Leningrad. Otdel. Mat. Inst. Steklov. (LOMI) 92 (1979) (Russian). MR 81h:46018

[C] D. Clark, One dimensional perturbations of restricted shifts, J. Anal. Math. 25 (1972), 169-91. MR 46:692

[D] G. David, Analytic capacity, Cauchy kernel, Menger curvature and rectifiability, Harmonic analysis and partial differential equations, Chicago Lectures in Math., Univ. of Chicago Press (1999). MR 2001a:30027

[F] O. Frostman, Sur les produits de Blaschke, Kungl. Fysiogr. Sällsk. i Lund Förh. 12, 15 (1942), 169-182. MR 6:262e

[G] J. B. Garnett, Bounded analytic functions, Pure and Applied Mathematics, 96, vol. 273, Academic Press, Inc. [Harcourt Brace Jovanovich, Publishers], New York-London, 1981. MR 83g:30037

[N] N. K. Nikolski, Treatise on the shift operator, Grundlehren der mathematischen Wissenschaften [Fundamental Principles of Mathematical Sciences], vol. 273, Springer-Verlag, Berlin-New York, 1986.

[NTV] F. Nazarov, S. Treil and A. Volberg, Weak type estimates and Cotlar inequalities for Calderon-Zygmund operators on nonhomogeneous spaces, J. Amer. Math. Soc. 12 (1999), 909-929. MR 99f:42035

[P1] A. Poltoratski, On the boundary behavior of pseudocontinuable functions, St. Petersburg Math. J. 5 (1994), 389-406.

[P2] A. Poltoratski, On the distributions of boundary values of Cauchy integrals, Proc. Amer. Math. Soc. 124 No 8 (1996), 2455-2463. MR 96j:30057

[P3] A. Poltoratski, Properties of Exposed Points in the Unit Ball of $H^{1}$, Indiana Univ. Math. J. 50 (2001), 1789-1806.

[P4] A. Poltoratski, The Krein spectral shift and rank one perturbations of spectra, Algebra i Analiz 10 (1998 No 5), 143-183 (Russian; English translation to appear in St. Petersburg Math. J.).

[P5] A. Poltoratski, Finite rank perturbations of singular spectra, Internat. Math. Res. Notices no. 9 (1997), 421-436. MR 98d:47035

[P6] A. Poltoratski, Equivalence up to a Rank One Perturbation, Pacific J. of Math. 194 (2000), 175-188. MR 2001j:47013

[S] D. Sarason, Sub-Hardy Hilbert Spaces in the unit disk, The University of Arkansas lecture notes in the mathematical sciences; v. 10, J. Wiley and Sons, New York, 1994. MR 96k:46039

[St] E. Stein, Harmonic Analysis, Princeton Univ. Press, 1993. MR 95c:42002

[T] Tolsa X., Littlewood-Paley theory and the T(1) theorem with non-doubling measures, Adv. Math. 164 (2001), 57-116.

Department of Mathemathes, Texas A\&M University, College Station, Texas 77843

E-mail address: alexeip@math.tamu.edu 